\title{
Etude de la dynamique spatiale et structure des forêts denses sèches reliques du Togo: implications pour une gestion durable des aires protégées
}

\author{
Kossi ADJONOU $^{1 *}$, Oyétoundé DJIWA ${ }^{2}$, Yendouhame KOMBATE ${ }^{2}$, \\ Adzo Dzifa KOKUTSE ${ }^{1}$ et Kouami KOKOU ${ }^{1}$ \\ ${ }^{1}$ Laboratoire de Botanique et Ecologie Végétale, Faculté des Sciences, Université de Lomé, BP : 1515, \\ Lomé (TOGO), Tel : (+228) 225-50-94, Fax: (+228) 221-85-95. \\ ${ }^{2}$ Direction de la Planification, Ministère de l'Environnement et des Ressources Forestières, 01 BP: 4825, \\ Lomé (TOGO), Tel (228) 22128 97, Fax (228) 2210333. \\ *Auteur correspondant, E-mail: kossiadjonou@hotmail.com
}

\section{RESUME}

$\mathrm{Au}$ Togo, les forêts denses sèches sont actuellement très menacées par les activités humaines et seulement quelques reliques se retrouvent sous forme d'îlots dans la plaine centrale en particulier dans les aires protégées. Cette étude recherche, à partir de l'analyse de la dynamique spatiale et des paramètres structuraux, des informations scientifiques fiables devant guider les orientations d'aménagement de ces types forestiers dans la Réserve de Faune d'Abdoulaye (RFA). L'étude de la dynamique spatiale de la couverture végétale de la RFA est réalisée à travers une analyse diachronique des images LANDSAT de trois âges différents (1972, 1991 et 2000). L'appréciation des caractéristiques structurales des îlots de forêts denses sèches est basée sur l'interprétation des données d'inventaire forestier. Ainsi, l'analyse de la dynamique de la végétation de cette réserve révèle une diminution des superficies des formations forestières entre 1972 et 2000 caractérisée par un taux annuel de régression de 3\% sur l'ensemble de la réserve. Au total, 69 espèces ligneuses ont été inventoriées dans les forêts denses sèches avec une densité moyenne de 681 tiges/ha, un diamètre moyen de $22,03 \pm 17,04 \mathrm{~cm}$ et une hauteur moyenne estimée à 13,33 $\pm 6,22 \mathrm{~m}$. Ces résultats constituent des bases scientifiques qui permettent de tester les indicateurs écologiques de gestion durable des forêts denses sèches reliques du Togo. Ils fournissent également des informations sur la mise en œuvre des directives pour l'amélioration des méthodes de gestion passée des aires protégées, basée sur la répression et le monopole du gouvernement.

(C) 2010 International Formulae Group. All rights reserved.

Mots clés : analyse diachronique, régression forestière, indicateurs écologiques, réserve de faune d'Abdoulaye, Togo.

\section{INTRODUCTION}

Les forêts denses sèches constituent l'un des écosystèmes d'intérêts écologique et biologique importants de par leur superficie (Trochain, 1957). Elles sont les plus exploitées et les plus menacées de toute la planète (Sahu et al., 2007). En Afrique, ces types de forêts ne sont actuellement représentés que par des vestiges plus ou moins dégradés, qui s'étendent de la Guinée Bissau à la République Centrafricaine, en zone soudanienne et dans le domaine zambézien (White, 1986). Ces formations végétales sont encore importantes à Madagascar (Bellefontaine, 1997). 
$\mathrm{Au} \mathrm{Togo,} \mathrm{les} \mathrm{forêts} \mathrm{denses} \mathrm{sèches}$ estimées à 243,75 km² dans les années 1975 (Vanpraet, 1980) sont actuellement très menacées par les activités humaines et leur superficie décroît continuellement. Seules quelques reliques se rencontrent sous forme d'îlots dans la plaine centrale et en particulier dans les aires protégées. La Réserve de Faune d'Abdoulaye (RFA), d'une superficie totale de 30000 ha, en renferme environ 8300 ha, réparties en taches de 500 à 600 ha (Vanpraet, 1980 ; Kokou et al., 2006 ; Djiwa, 2008). Ces taches sont très représentatives des forêts denses sèches du Togo, l'un des pays d'Afrique de l'Ouest à très faible couverture forestière; celles situées hors des aires protégées étant quasiment converties en champs et sites d'habitation.

Face aux menaces permanentes de dégradation, le Ministère de l'Environnement et des Ressources Forestières a initié un programme de réhabilitation de la plupart des aires protégées prioritaires du Togo dont celle de la RFA. Dans cette dernière, le programme devra aboutir à l'élaboration d'un plan d'aménagement visant une conservation et une gestion durable des fragments de forêts de cette réserve. Pour atteindre les objectifs du programme, la conception et l'élaboration de ce plan d'aménagement nécessitent des bases scientifiques fiables sur lesquelles doivent s'appuyer les activités d'aménagement à court, à moyen ou à long terme.

En vue d'aider les gestionnaires, la présente étude se propose de mettre à leur disposition des connaissances scientifiques qui prennent en compte les indicateurs écologiques comme la dynamique spatiale et structurale des fragments de forêts denses sèches dans le plan d'aménagement de la RFA. Il s'agit plus spécifiquement: 1)d'étudier la dynamique spatiale des formations végétales suite à l'occupation humaine lors des troubles socio-politiques des années 90 et, 2)- d'analyser les caractéristiques structurales des fragments de forêts denses sèches afin de connaître la santé actuelle de la forêt. Ces informations sont nécessaires aux orientations d'aménagement et de gestion durable de ces îlots de forêts rares et menacés au Togo.

\section{MATERIEL ET METHODES \\ Zone d'étude}

Classée par le décret $n^{\circ}$ 391-51/EF du 7 juin 1951, la RFA a pour objectif la conservation in situ de la biodiversité (la faune, la flore, les écosystèmes forestiers et les paysages). Elle est localisée dans la préfecture de Tchamba dans la plaine centrale du Togo, entre les latitudes $8^{\circ} 33^{\prime}$ et $8^{\circ} 47$ Nord et les longitudes $1^{\circ} 15^{\prime}$ et $1^{\circ} 27^{\prime}$ Est (Figure 1). Elle couvrait avant 1970 une superficie de 30000 ha.

La plaine centrale présente un paysage en interfluve (avec une altitude comprise entre 250 et $350 \mathrm{~m}$ ) et repose sur le socle cristallin granito-gneissique de l'unité structurale de la plaine bénino-togolaise. Elle jouit d'un climat tropical de type guinéen caractérisé par une saison pluvieuse d'avril à octobre et une saison sèche de novembre à mars. La pluviosité varie entre 1200 et $1300 \mathrm{~mm} / \mathrm{an}$. L'humidité relative varie entre 60 et $80 \%$. L'évaporation est d'environ $1600 \mathrm{~mm} / \mathrm{an}$. Les températures mensuelles varient entre 20 et 32 ${ }^{\circ} \mathrm{C}$ (avec une moyenne mensuelle de $26,5^{\circ} \mathrm{C}$ ). Les sols dominants sont de types ferrallitiques mais de faciès différents, peu profonds avec une texture limono-argileuse de couleur rouge présentant des concrétions ferrugineuses. Les sols hydromorphes se rencontrent le long des cours d'eau.

\section{Collecte des données \\ Données satellitaires}

L'étude de la dynamique spatiale de la végétation de la RFA est basée sur l'interprétation d'images satellitaires par une analyse diachronique des images de trois âges différents. Les images satellitaires utilisées sont LANDSAT MSS WRS (p207/r054) du 11 octobre 1972, LANDSAT ETM (p192/r054) du 10 janvier 1991 et LANDSAT ETM+ (p192/r054) du 04 décembre 2000. L'image de 1991 a été choisie afin de déterminer la situation de l'occupation du sol peu avant l'envahissement de la période 19902003 de manière à ressortir les dégradations 


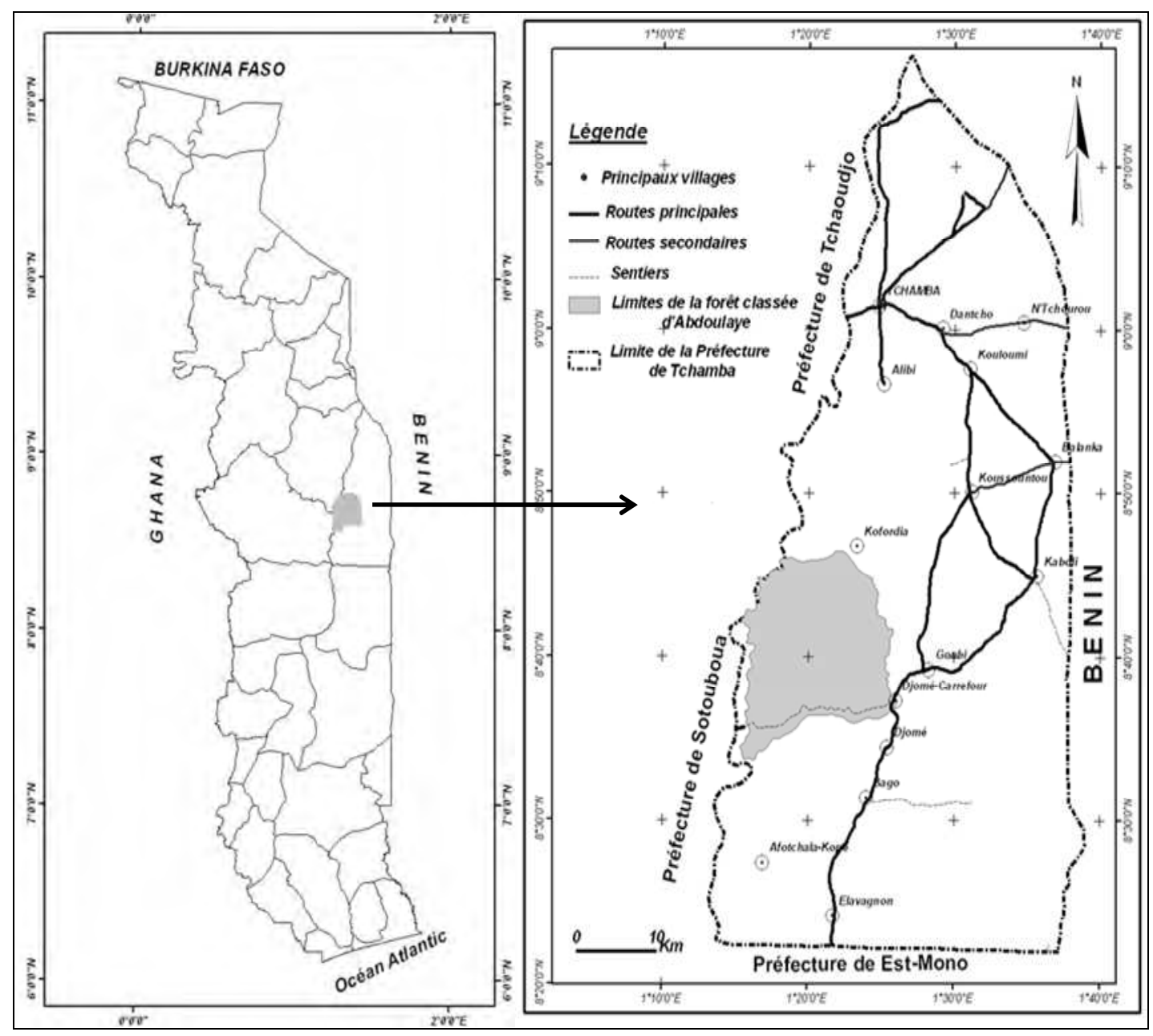

Figure 1: Localisation de la zone d'étude (Réserve de Faune d'Abdoulaye).

directement imputables à l'installation des populations dans la RFA. L'image de 2000 est la plus récente disponible pour la zone d'étude. Un GPS (Global Positioning System) a été utilisé pour localiser les points d'échantillonnage pour la reconnaissance et le contrôle de terrain.

\section{Inventaires forestiers}

L'étude des caractéristiques structurales des forêts denses sèches (FDS) est réalisée grâce à l'analyse des données d'inventaires réalisés dans ces types de forêts. Dans la RFA, les inventaires ont été réalisés uniquement dans les fragments de FDS dans 52 placeaux de forme carrée de $625 \mathrm{~m}^{2}(25 \mathrm{~m}$ x $25 \mathrm{~m}$ ), soit 3,25 ha sur 4700 ha (taux d'échantillonnage $=0,07 \%$ ). Cette méthode d'inventaire avec des placeaux de $625 \mathrm{~m}^{2}$ comme unité d'échantillonnage a été déjà utilisée par de nombreux auteurs dans des études similaires (Hall et Swaine, 1981; Adou Yao et al., 2005; Zapfack, 2005 ; Adjonou et al., 2009). Ils consistent à mesurer la circonférence et la hauteur totale des ligneux. La circonférence a été mesurée avec un mètreruban à $1,30 \mathrm{~m}$ du sol pour les arbres (hauteur $>5 \mathrm{~m}$ ) et à $0,25 \mathrm{~m} \mathrm{du}$ sol pour les arbustes (hauteur $<5 \mathrm{~m}$ et $>1 \mathrm{~m}$ ) (Kokou, 1998). Leur hauteur totale a été mesurée avec un relascope de Bitterlich. Le potentiel de régénération a été évalué par le biais de cinq petits placeaux de $25 \mathrm{~m}^{2}$ ( $5 \mathrm{~m}$ x $5 \mathrm{~m}$ ) délimités à l'intérieur de chaque placeau d'échantillonnage. Au total, 260 petits placeaux (soit environ 0,65 ha) ont été installés. Les semis naturels, rejets de souche, 
drageons (et d'éventuelles marcottes terrestres) dont la circonférence est inférieure à $10 \mathrm{~cm}$ ont été pris en compte (Sokpon et al., 2006). Parfois des excavations à la base des tiges sont nécessaires pour vérifier et/ou pour faire la différence entre rejets de souches et drageons.

\section{Analyse et traitement des données}

La méthode utilisée pour l'analyse des images satellitaires s'appuie sur la technique d'interprétation visuelle des images (Koné et al., 2007). A partir de la carte topographique au 1/200 000 de la région centrale du Togo, géo-référencée par rapport à l'ellipsoïde WGS 84 et le fuseau 31 Nord, on a procédé à l'extraction de la zone d'étude par numérisation de son contour. Le logiciel utilisé pour ce faire est ARCGIS version 9.1. Les images sont ensuite exportées dans le logiciel ERDAS-Imagine où l'analyse visuelle et la technique de classification automatique des images satellites sur la base de l'algorithme du maximum de vraisemblance est réalisée (Mama et Oloukoi, 2003). L'analyse visuelle des images par composition colorée RVB-432 a permis dans un premier temps d'émettre des hypothèses d'occupation du sol. Les classifications non-supervisée et supervisée, combinées à une vectorisation automatique ont permis de générer des informations sur la couverture végétale. Les types de formations végétales adoptés pour la classification sont ceux retenus pour l'Afrique de l'Ouest (FAO, 1981).

Pour s'assurer de la précision de la classification de l'occupation du sol réalisée à partir des données de 2000, une méthode basée sur l'évaluation des points de contrôle a été retenue. Pour ce faire, 100 points de contrôle à raison de 20 points par type de formation ont été retenus. Cette méthode consiste à vérifier sur le terrain, les points précédemment identifiés avant la mission de terrain pour chacune des classes de l'occupation du sol et à déterminer le pourcentage des points vérifiés qui correspondent à ceux définis au préalable (Mama et Oloukoi, 2003). Le pourcentage de points de terrain qui s'accordent avec ceux de la carte détermine le degré de précision de l'interprétation de l'image satellitaire. Une matrice de confusion a été construite à cet effet pour rendre compte des résultats (Tableau 1). L'indice de Kappa a été évalué à 60\% dans ERDAS. Lorsque l'indice de Kappa évalué dans les opérations de classification est compris entre 50 et $75 \%$, la classification adoptée est valable et les résultats peuvent être judicieusement utilisés (Pointus, 2000). Pour chaque unité d'occupation du sol, le taux d'évolution annuel est calculé selon la formule ci-après: $T_{\text {annuel }}=\frac{S_{i 2000}-S_{i 1991}}{d \times 100}$ avec $\mathrm{S}_{\mathrm{i} 2000}$ et $\mathrm{S}_{\mathrm{i} 1991}$ les superficies des différents types de formation respectivement en 2000 et en 1991; d étant la durée en années de la période 1991 à 2000 qui est 9 ans dans ce cas.

L'analyse floristique a permis de dresser la liste des espèces recensées et de les regrouper par famille. La nomenclature utilisée est celle de Lebrun and Stock (19911997). Les caractéristiques forestières sont appréciées directement par calcul arithmétique pour la densité, la hauteur totale moyenne et le diamètre moyen. La densité des arbres a été évaluée en nombre de pieds par hectare pour les arbres dont la circonférence à $1,30 \mathrm{~m} \geq 10$ $\mathrm{cm}$. La surface terrière (en $\mathrm{m}^{2} / \mathrm{ha}$ ) a été calculée suivant la formule $\mathrm{G}=\Sigma \pi \mathrm{d}^{2} / 4$ (où $\mathrm{d}$ est le diamètre à $1,30 \mathrm{~m}$ ). Le volume des arbres est calculé par la formule volumétrique: $\mathrm{V}=\mathrm{F} \times \mathrm{G} \times \mathrm{H}$, (avec $\mathrm{F}=$ coefficient de forme; $\mathrm{G}=$ surface terrière de l'arbre; $\mathrm{H}=$ hauteur totale de l'arbre; la valeur 0,5 a été choisie pour F). Cette valeur est largement utilisée en milieux tropicaux pour des arbres dont le diamètre à hauteur de poitrine (dbh) est supérieur à $7 \mathrm{~cm}$; mais pour des diamètres plus petits, Lopez (2004) a utilisé un coefficient de 0,6 . La relation allométrique qui existe entre le diamètre à $1,30 \mathrm{~m}$ et la hauteur totale est un indicateur de croissance des arbres; elle est exprimée par une régression logarithmique d'équation $\mathrm{Y}=\mathrm{a} \mathrm{x} \ln (\mathrm{d})+\mathrm{b}$ (où $\mathrm{Y}=$ hauteur totale, $\mathrm{d}=$ diamètre à $1,30 \mathrm{~m}$ du sol, a et b sont des constantes). La densité de la régénération est estimée et l'importance relative de chaque mode de régénération est 
exprimée en pourcentage. L'analyse des données structurales combinées aux données floristiques permet de calculer l'Indice de Valeur d'Importance (IVI). L'IVI est utilisé pour évaluer la prépondérance spécifique en forêts tropicales. Il se calcule selon la formule de Curtis et Macintosh (1950): IVI = Dominance relative (espèce) + Densité relative (espèce) $^{+}$Fréquence relative (espèce), où:

- la dominance relative d'une espèce est le quotient de son aire basale avec l'aire basale totale de toutes les espèces multiplié par 100 ;

- la densité relative d'une espèce est le rapport de sa densité absolue au total des densités absolues de toutes les espèces multiplié par 100;

- la fréquence relative d'une espèce est le rapport de sa fréquence spécifique par le total des fréquences spécifiques de toutes les espèces multiplié par 100.

\section{RESULTATS}

Dynamique de l'occupation du sol de la RFA entre 1972 et 2000

Il ressort de l'analyse des points de contrôle pour la classification que sur 100 points contrôlés, 75 correspondent aux types de formations auxquels ils avaient été attribués. Cela génère une précision moyenne de $75 \%$ pour l'ensemble de la zone d'étude (Tableau 1).

En 1972, deux types de formations caractérisent la RFA. Il s'agit des formations forestières (FDS et forêts galeries $=27 \%$ ), les formations de savanes (savane boisée $(31 \%)$ et savane arborée (42\%)). A partir de 1991, on observe (Figures 2):

- $\quad$ une diminution des superficies des FDS et de la savane boisée,

- une très faible variation de la superficie des savanes arborées,

- une réaffectation d'une partie de la RFA en savane arbustive.

De l'analyse des images satellitaires de 2000, la RFA est constituée de quatre types d'occupation du sol. Il s'agit de:
- FDS et galeries forestières: elles sont sous forme de mosaïque, essentiellement localisées dans la partie sud-est de la réserve, le long de la rivière Ogou et de ses principaux affluents et occupent environ $10 \%$ de la superficie totale de la RFA ;

- savanes boisées/arborées: elles constituent la formation savanicole la plus importante et occupent près de $65 \%$ de la superficie totale de la réserve ;

- savanes arbustives : elles occupent près de $15 \%$ de la superficie totale de la réserve et est principalement localisée au centre et dans la partie ouest de la réserve sous forme d'ilots d'environ 10 à 100 ha ;

- les jachères vieilles d'environ 5 à 10 ans, occupent près de $10 \%$ de la superficie totale de la réserve. Elles sont principalement localisées dans la partie sud le long de la piste traversant la réserve dans la direction EstOuest.

D’une manière générale, les changements d'affectation et d'utilisation des terres entre 1972 et 2000 sont caractérisés par une dimunition des superficies des formations forestières et des savanes arborées au profit de la savane arbustive et des zones de culture.

L'interprétation des images satellites révèle un taux annuel de régression de $3 \%$ sur l'ensemble de la RFA (Tableau 2, Figure 3). Plus spécifiquement, on observe une augmentation du taux annuel des savanes arbustives $(1,77 \%)$ et des zones de culture et des jachères $(1,12 \%)$ et une baisse du taux annuel des FDS et des galeries forestières ($1,29 \%)$ et des savanes arborées $(-1,70 \%)$. Cette régression des superficies forestières s'explique surtout par des actions anthropiques (intrusion des populations dans la forêt pour l'agriculture, feux de brousse criminels, coupes frauduleuses, etc) et probablement par les causes climatiques (faible pluviométrie enregistrée dans la région (1200 à $1300 \mathrm{~mm} / \mathrm{an}$ ) et longue période de sécheresse des années 1968-1973 et 1980). 


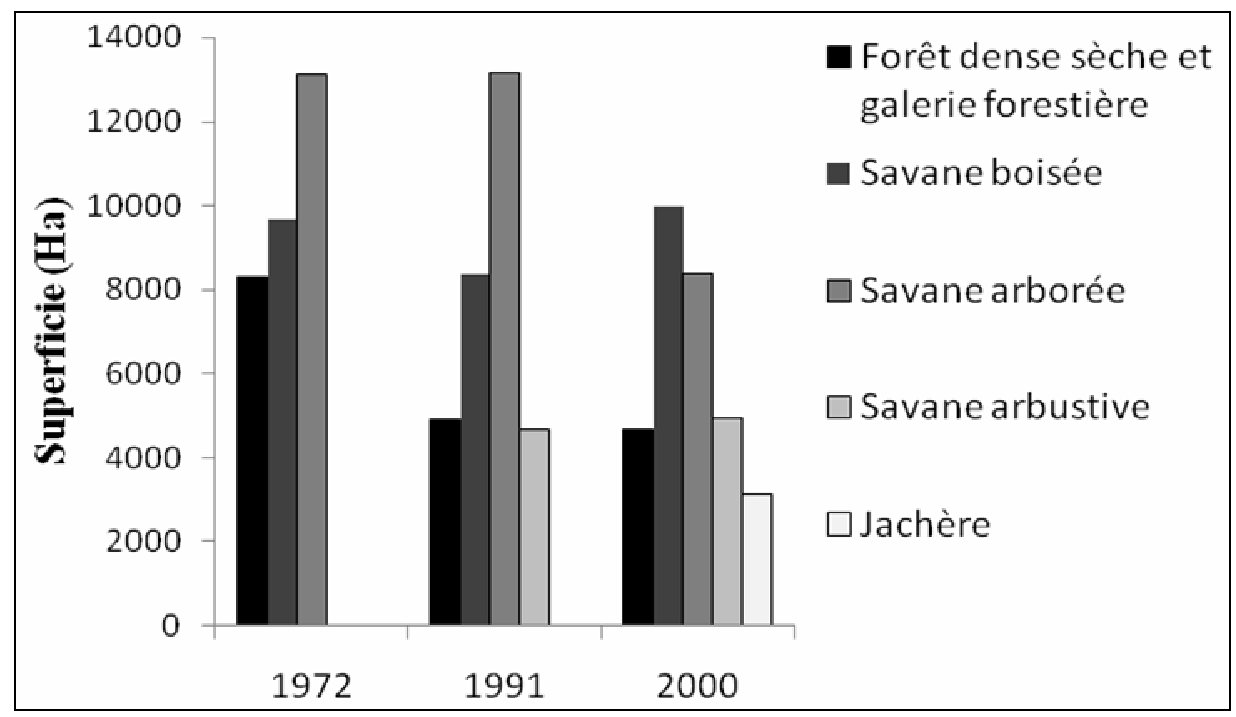

Figure 2: Evolution de la superficie de la couverture végétale dans la RFA de 1972 à 2000.

Tableau 1 : Matrice de confusion pour l'estimation de la précision de la classification.

\begin{tabular}{lcccccc}
\hline Type d'occupation & $\begin{array}{c}\text { Forêt dense } \\
\text { sèche }\end{array}$ & $\begin{array}{c}\text { Savane. } \\
\text { boisée }\end{array}$ & $\begin{array}{c}\text { Savane } \\
\text { arborée }\end{array}$ & $\begin{array}{c}\text { Savane } \\
\text { arbustive }\end{array}$ & Jachère & Total \\
\hline Forêt dense sèche & $\mathbf{1 8}$ & 2 & & & & 20 \\
Savane boisée & 2 & $\mathbf{1 6}$ & 1 & & & 19 \\
Savane arborée & & 2 & $\mathbf{1 5}$ & 1 & & 18 \\
Savane arbustive & & & 4 & $\mathbf{1 3}$ & 7 & 24 \\
Jachère & & 20 & 20 & 20 & 20 & $\mathbf{7 5}$ \\
Total & 20 & & & 6 & $\mathbf{1 3}$ & 19 \\
\hline
\end{tabular}

Tableau 2: Taux d'évolution annuelle des unités d'occupation du sol

\begin{tabular}{lccc}
\hline \multirow{1}{*}{ Unités d'occupation du sol } & \multicolumn{3}{c}{ Taux annuel d'évolution en \% } \\
\cline { 2 - 4 } & 1972 à 1991 & 1991 à 2000 & $\mathbf{1 9 7 2}$ à 2000 \\
\hline Forêt dense sèche et galeries forestières & $-1,78$ & $-0,26$ & $\mathbf{- 1 , 2 9}$ \\
Savane boisée & $-0,69$ & 1,78 & $\mathbf{0 , 1 1}$ \\
Savane arborée & 0,01 & $-5,31$ & $\mathbf{- 1 , 7 0}$ \\
Savane arbustive & 2,45 & 0,33 & $\mathbf{1 , 7 7}$ \\
Zones de culture/jachères & 0,00 & 3,47 & $\mathbf{1 , 1 2}$ \\
\hline
\end{tabular}


K. ADJONOU et al. /Int. J. Biol. Chem. Sci. 4(1): 168-183, 2010

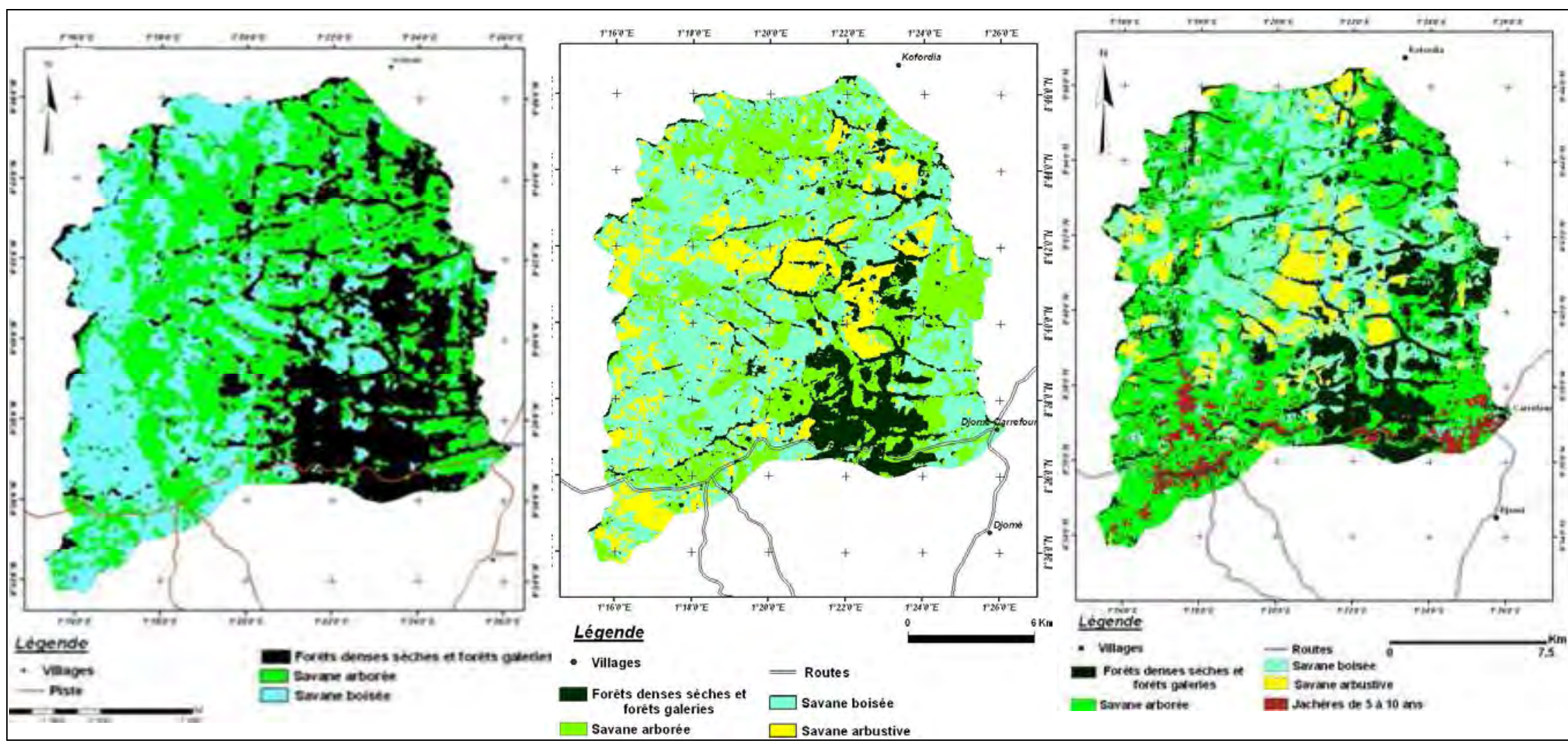

Image de 1972

Image de 1991

Image de 2000

Figure 3: Carte de la dynamique de l'occupation du sol dans la Réserve de Faune d'Abdoulaye entre 1972 et 2000. 


\section{Caractéristiques structurales des FDS de la} RFA

\section{Structure des peuplements ligneux des FDS de la RFA}

$\mathrm{Au}$ total, 69 espèces ligneuses ont été recensées dans 52 relevés réalisés. Ces espèces sont réparties en 62 genres et 26 familles. Les arbres représentent $68,1 \%$ et les arbustes $31,9 \%$. Les familles les mieux représentées en nombre d'espèces sont les Légumineuses (16 espèces), les Rubiacées (8 espèces), les Sapotacées (7 espèces) et les Moracées (4 espèces).

Dans les îlots de forêts denses sèches de la RFA, la densité des arbres est évaluée à 681 pieds/ha et la surface terrière est de 41,46 $\mathrm{m}^{2} /$ ha avec un bio-volume ligneux total évalué à $319,05 \mathrm{~m}^{3} /$ ha (Tableau 3). Le diamètre moyen de la population ligneuse est évalué à $22,03 \pm 17,04 \mathrm{~cm}$ (Tableau 3). Parmi les espèces de bois d'œuvre dont le diamètre peut atteindre $60 \mathrm{~cm}$ et plus, on peut citer Anogeissus leiocarpus, Antiaris africana, Ceiba pentadra, Celtis zenkeri, Diospyros mespiliformis, Khaya senegalensis, etc.

La répartition des individus ligneux par classes de diamètre présente l'allure d'une courbe dissymétrique centrée sur la classe de diamètre 10 à $20 \mathrm{~cm}$ (Figure 4) traduisant ainsi la prédominance des individus de petit diamètre. Cette distribution s'ajuste mieux avec une fonction polygonale dont l'équation est : $Y=-3,2348 x^{4}+57,16 x^{3}-342,42 x^{2}+$ $753,77 x-309\left(R^{2}=0,9674\right)$. Par ailleurs, la répartition de la surface terrière par classe de diamètre montre que les individus de grand diamètre $(50 \mathrm{~cm}$ et plus $)$ tels que $A$. leiocarpus, Antiaris africana, Ceiba pentadra, Celtis zenkeri, Cola gigantea et Erythrophleum suaveolens cumulent l'essentiel de la surface terrière totale.

$\mathrm{La}$ hauteur totale moyenne du peuplement est évaluée à 13,33 $\pm 6,22 \mathrm{~m}$. La répartition des ligneux par classes de hauteur totale est illustrée par la Figure 5 qui présente une distribution en dôme centrée sur les classes de hauteur comprises entre 8 et $12 \mathrm{~m}$. Sa courbe représentative s'ajuste mieux à une fonction polygonale dont l'équation est : $\mathrm{Y}=$ $0,5987 x^{3}-14,019 x^{2}+90,928 x-75,879\left(R^{2}=\right.$
0,846). Il faut noter que dans la RFA, on retrouve une proportion non négligeable d'individus ligneux dont la hauteur totale peut avoisiner $15 \mathrm{~m}$ et plus.

La relation allométrique exprimant la croissance entre le diamètre à $1,30 \mathrm{~m}$ et la hauteur totale est illustrée par la Figure 6. Cette relation s'exprime par une fonction logarithmique dont l'équation est: $\mathrm{Y}=8,0332$ $\ln (x)-9,5823\left(R^{2}=0,8091\right)$. Ce graphique indique que la hauteur maximale des arbres se stabilise autour de $30 \mathrm{~m}$ pour un diamètre de $120 \mathrm{~cm}$. Dans cette forêt, très peu d'arbres (soit environ $0,4 \%$ ) ont une hauteur qui excède cette hauteur maximale et seulement $0,3 \%$ des arbres inventoriés ont un diamètre supérieur à 120 cm.

\section{Potentiel de régénération}

La densité moyenne de la régénération naturelle, toutes espèces confondues, dans les îlots de forêts denses sèches de la RFA est évaluée à 5508 tiges/ha pour 57 espèces inventoriées. Cette régénération est essentiellement dominée par trois espèces à savoir Pouteria alnifolia, Lecaniodiscus cupanioides et Holarrhena floribunda qui ont respectivement une densité de population évaluée à 1346 tiges/ha, 892 tiges/ha et 698 tiges/ha. Certaines des espèces «clés» recensées dans ces types de forêts possèdent un bon potentiel de régénération. Parmi ces espèces, on peut citer A. africana (161 tiges/ha), D. mespiliformis (130 tiges/ha), Sterculia tragacantha (64 tiges/ha). Par contre, d'autres espèces non moins importantes, présentent une faible capacité de régénération. Il s'agit des espèces telles que $A$. leiocarpus (17 tiges/ha), K. senegalensis (9 tiges/ha), Aubrevillia kerstingii (8 tiges/ha), Pterocarpus erinaceus (3 tiges/ha) et Afzelia africana (2 tiges/ha).

La répartition des espèces par modes de régénération, montre que ces espèces se régénèrent essentiellement par semis $(82,04 \%$ des jeunes sujets inventoriés), faiblement par rejets de souches $(17,66 \%)$ et rarement par drageonnage $(0,31 \%)$. Les observations dans ces îlots forestiers ont montré que $P$. alnifolia, 
Tableau 3: Importance de quelques espèces dans la Réserve de Faune d'Abdoulaye.

\begin{tabular}{lccccc}
\hline NOMS SCIENTIFIQUES & FAMILLE & DER & DOR & FRE & IVI \\
\hline Diospyros mespiliformis. & Ebenaceae & 24,3 & 14,1 & 7,8 & 38,4 \\
Anogeissus leiocarpus & Combretaceae & 8,8 & 27,4 & 7,0 & 36,2 \\
Pouteria alnifolia & Sapotaceae & 9,0 & 7,6 & 7,4 & 16,5 \\
Cola gigantea & Sterculiaceae & 5,7 & 9,9 & 4,6 & 15,6 \\
Mimusops kummel & Sapotaceae & 8,8 & 4,6 & 3,0 & 13,4 \\
Ceiba pentadra & Bombacaceae & 0,5 & 7,7 & 1,6 & 8,2 \\
Bequaertiodendron oblanceolatum & Sapotaceae & 5,8 & 1,2 & 4,2 & 6,8 \\
Manilkara obovata & Sapotaceae & 4,0 & 2,6 & 5,9 & 6,6 \\
Erythrophleum suaveolens & Fabaceae & 1,9 & 4,6 & 4,3 & 6,5 \\
Holarrhena floribunda & Apocynaceae & 2,8 & 1,6 & 3,8 & 4,4 \\
Lecaniodiscus cupanioides & Sapindaceae & 3,0 & 0,7 & 4,2 & 3,6 \\
Holoptelea grandis & Ulmaceae & 1,3 & 2,3 & 1,1 & 3,5 \\
Pachystela brevipes & Sapotaceae & 2,9 & 0,5 & 0,6 & 3,5 \\
Polysphaeria arbuscula & Rubiaceae & 3,2 & 0,3 & 4,0 & 3,4 \\
Celtis zenkeri & Ulmaceae & 1,6 & 1,5 & 3,7 & 3,1 \\
Zanthoxylum zanthoxyloides & Rutaceae & 1,1 & 1,5 & 2,2 & 2,6 \\
Psydrax horizontale & Rubiaceae & 1,3 & 0,9 & 2,4 & 2,7 \\
Antiaris africana & Moraceae & 0,8 & 1,4 & 2,2 & 2,2 \\
Khaya senegalensis & Meliaceae & 0,9 & 0,8 & 2,1 & 1,7 \\
Autres & & & & & \\
\hline TOTAL & & 12,3 & 8,8 & 27,9 & 21,1 \\
\hline DER Densie Reluie; DOR & $\mathbf{1 0 0}$ & $\mathbf{1 0 0}$ & $\mathbf{1 0 0}$ & $\mathbf{2 0 0}$ \\
\hline
\end{tabular}

$\overline{D E R}=$ Densité Relative $;$ DOR $=$ Dominance Relative $; F R E=$ Fréquence Relative, $I V I=$ Indice de Valeur d'Importance .

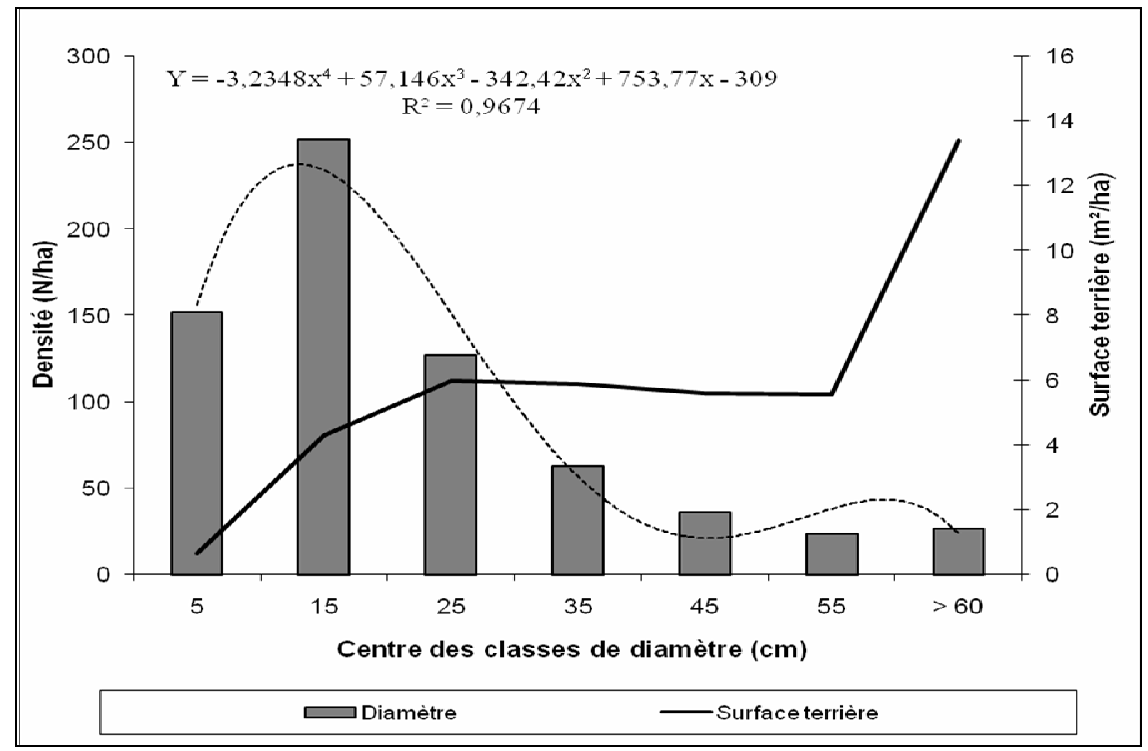

Figure 4: Distribution des arbres et de leur surface terrière par classes de diamètre. 


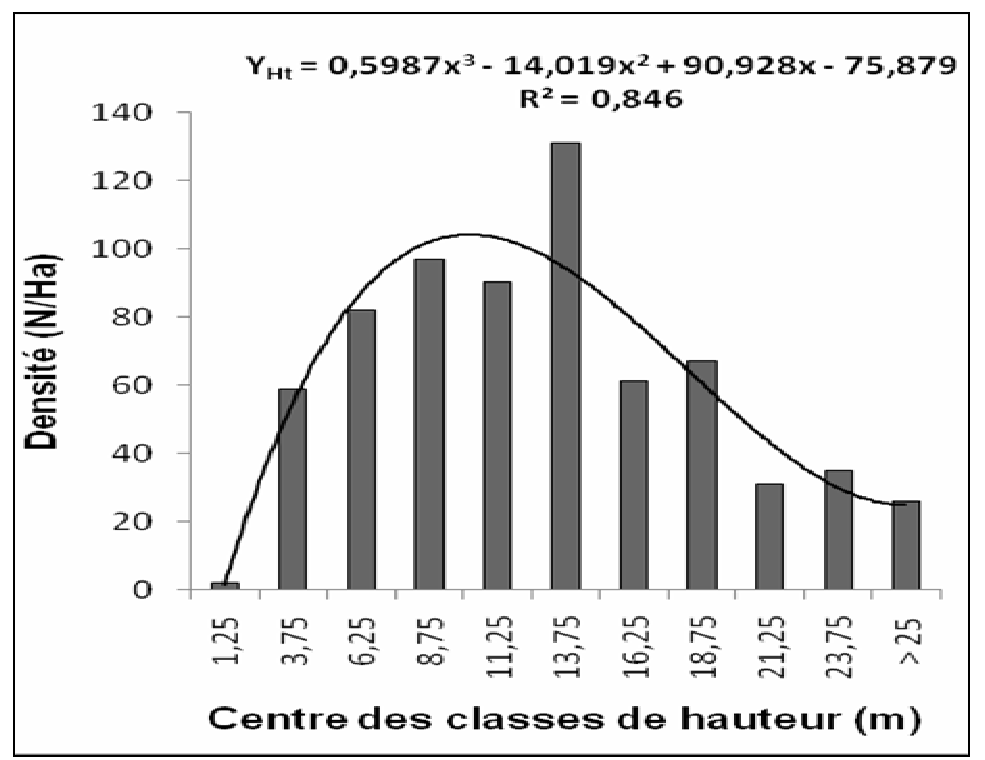

Figure 5 : Distribution des arbres par classes de hauteur totale.

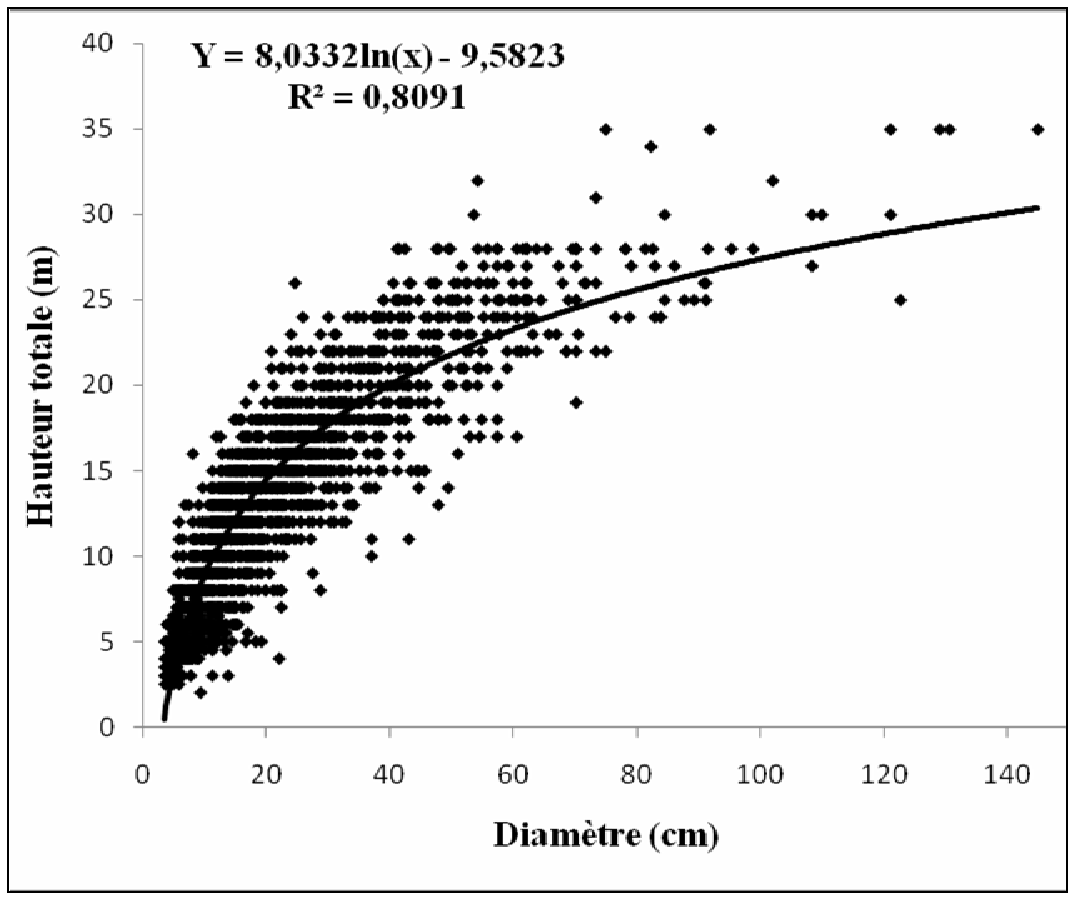

Figure 6: Corrélation entre la hauteur totale et le diamètre à 1,30 m.

une espèce de la strate arborescente moyenne, régénère à la fois par semis, par rejets de souches et par drageons. La plupart des espèces qui régénèrent par semis, le font également par rejets de souche étant donné que ce dernier mode de régénération est souvent induit par des actions exogènes. Dans les conditions de cette étude, les modes de régénération par marcottage terrestre et par bouturage n'ont pas été observés.

Parmi les espèces inventoriées dans les FDS de la RFA, cinq espèces (Diospyros mespiliformis, Anogeissus leiocarpus, Pouteria alnifolia, Cola gigantea et Mimusops 
kummel), cumulent près des $2 / 3(78,9 \%)$ du total de l'IVI (Tableau 4). Les autres espèces ne représentent qu'environ $1 / 3(21,1 \%)$ de la valeur totale des IVI avec une densité relative cumulée égale à $0,3 \%$, une dominance relative cumulée égale à $0,8 \%$ et une fréquence relative cumulée égale à $27,7 \%$. D . mespiliformis et A. leiocarpus sont les deux principales espèces des FDS de la RFA, avec des IVI respectivement évalués à 38,4 et 36,2 (Tableau 4).

\section{Espèces «clés» de la RFA : Diospyros mespiliformis et Anogeissus leiocarpus}

Les îlots de FDS de la RFA sont dominés par deux principales espèces à savoir D. mespiliformis et A. leiocarpus. Ces deux espèces présentent respectivement une densité de population évaluée à 168 tiges/ha et 61 tiges/ha (Tableau 5).

La répartition des deux espèces par classes de diamètre montre deux situations différentes. D. mespiliformis présente une structure en cloche dissymétrique avec une prédominance des individus de petit diamètre (classe 10-20 $\mathrm{cm}$ ) traduisant une bonne dynamique de la population. Par contre, A. leiocarpus montre une distribution en forme de «J» avec une prédominance des individus de grand diamètre (diamètre $>50 \mathrm{~cm}$ ) traduisant le caractère vieillissant de ces peuplements (Figure 7).

La structure verticale des deux principales espèces ne montre pas la même distribution. D. mespiliformis a une distribution en cloche symétrique centrée sur la classe de hauteur totale 10 à $15 \mathrm{~m}$ avec certains individus dont la hauteur peut atteindre $25 \mathrm{~m}$ alors que A. leiocarpus montre une distribution en cloche dissymétrique centrée sur la classe de hauteur 25 à $30 \mathrm{~m}$.

\section{DISCUSSION}

De nombreux critères et indicateurs (C\&I) ont été proposés pour assurer la gestion forestière durable dans le monde (OAB-OIBT, 2003; Mendoza and Prabhu, 2003; Prabhu et al., 2005). Cependant, leur validité scientifique reste incertaine en raison de la spécificité des sites, des paysages, des régions, des types forestiers, etc. (Miles, 2002; Lindenmayer et al., 2006). Cette étude permet de tester les indicateurs écologiques de gestion durable sur les forêts denses sèches de la RFA. En effet, l'analyse diachronique de la couverture végétale de la RFA a permis d'établir un zonage ou unités d'aménagement constituées de forêts denses sèches et galeries forestières (environ 10\% de la superficie totale), de savanes boisées/arborées (65\%), de savanes arbustives (15\%) et de jachères (10\%) (Principe 2, Critère 2.1; Boyle et al., 1998). Elle a permis aussi d'établir le taux de dégradation annuelle des forêts denses sèches et galeries forestières qui est de 1,29\% par an. Pendant ce temps, les savanes et les cultures s'élargissent de $1,77 \%$. Les causes de la dégradation des forêts sont connues; Kokou et al. (2006) ont montré qu'elles sont d'origine anthropique (feux et agriculture et exploitation forestière). Mais également, l'assèchement du climat ces dernières années peut être évoqué ; la RFA se situe dans une zone écologique sèche qui ne bénéficie pas de la pluviosité minimale devant permettre la reconstitution spontanée des formations végétales (Vanpreat, 1980). Les sécheresses des années 1968-1973 et des années 1980 auraient également joué un rôle important dans la régression de la couverture forestière (Barbier, 2004).

L'appréciation des paramètres structuraux de ces îlots de forêts denses sèches a permis d'élaborer un diagnostic préliminaire sur leur potentiel forestier; 69 espèces végétales ligneuses y ont été relevées avec une densité moyenne de 681 tiges/ha sur l'ensemble de la réserve. Cette densité de population ligneuse montre que les îlots de forêts denses sèches de la RFA disposent encore d'un important potentiel ligneux malgré les diverses pressions que ces formations forestières ont subi. Cette valeur de la densité correspond à celle d'autres auteurs qui estiment que la densité moyenne des arbres de $\mathrm{dbh}>10 \mathrm{~cm}$ sous les tropiques se situent entre 276 et 905 tiges/ha, (Sundarapandian and Swamy, 1997; Ghate et al., 1998). L'analyse de la distribution des individus par classes de diamètre révèle une 
Tableau 4: Principales caractéristiques des FDS de la RFA.

\begin{tabular}{lc}
\hline Paramètres structuraux & Valeurs \\
\hline Densité des arbres $(\mathrm{N} / \mathrm{ha})$ & 681,23 \\
Diamètre moyen $(\mathrm{cm})$ & $22,03 \pm 17,04$ \\
Hauteur totale moyenne $(\mathrm{m})$ & $13,33 \pm 6,22$ \\
Hauteur moyenne de fût $(\mathrm{m})$ & $5,10 \pm 3,60$ \\
Surface terrière $\left(\mathrm{m}^{2} / \mathrm{ha}\right)$ & 41,46 \\
Biomasse ligneux $\left(\mathrm{m}^{3} / \mathrm{ha}\right)$ & 140,92 \\
\hline \multicolumn{2}{c}{ Les valeurs du tableau expriment la moyenne \pm l'écart type $;$ N/ha: nombre de pieds/ha. }
\end{tabular}

Tableau 5: Principales caractéristiques forestières des deux espèces dominantes.

\begin{tabular}{lcc}
\hline Caractéristiques forestières & Diospyros mespiliformis & Anogeissus leiocarpus \\
\hline Densité de la population $(\mathrm{N} / \mathrm{ha})$ & 168 & 61 \\
Diamètre moyen $(\mathrm{cm})$ & $18,36 \pm 10,31$ & $44,37 \pm 19,86$ \\
Hauteur totale moyenne $(\mathrm{m})$ & $13,08 \pm 4,66$ & $22,01 \pm 5,36$ \\
\hline Les valeurs du tableau expriment la moyenne + l'écart type $:$ N/ha: nombre de pieds $/$ ha &
\end{tabular}

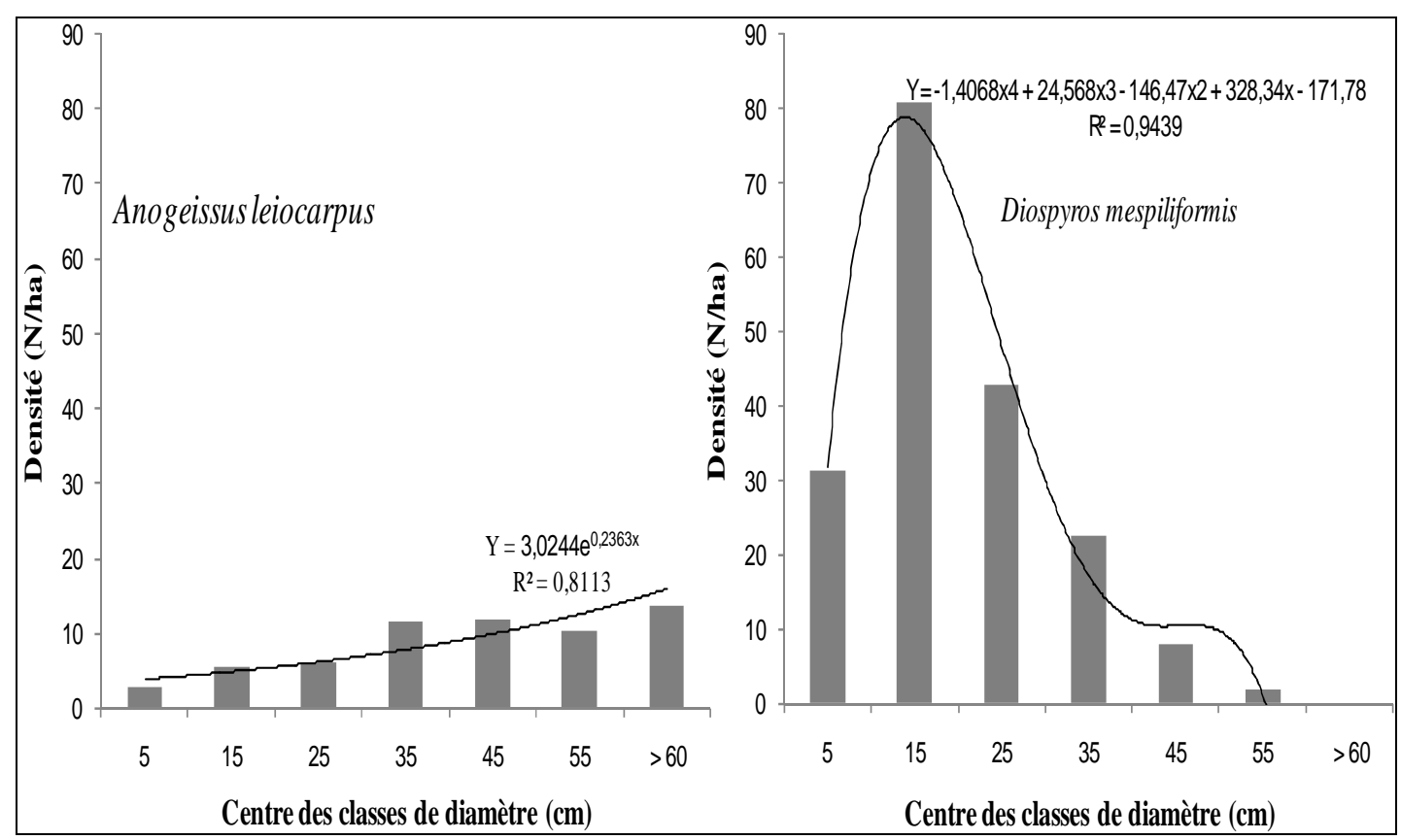

Figure 7 : Structure diamétrique des populations de D. mespiliformis et A. leiocarpus.

répartition dissymétrique traduisant la prédominance des individus de petit diamètre. Cette distribution a été également décrite par Rasatatsihoarana (2007) dans les mêmes types forestiers dans le Menabe Sud à Madagascar. Cette distribution indique que ces îlots forestiers présentent une dynamique régulière dénotant une régénération constante dans le temps (Rasatatsihoarana, 2007) et caractérise une population ligneuse s'entretenant entreelle (Fongnzossie et al., 2008). La prédominance des jeunes plantes influencerait 


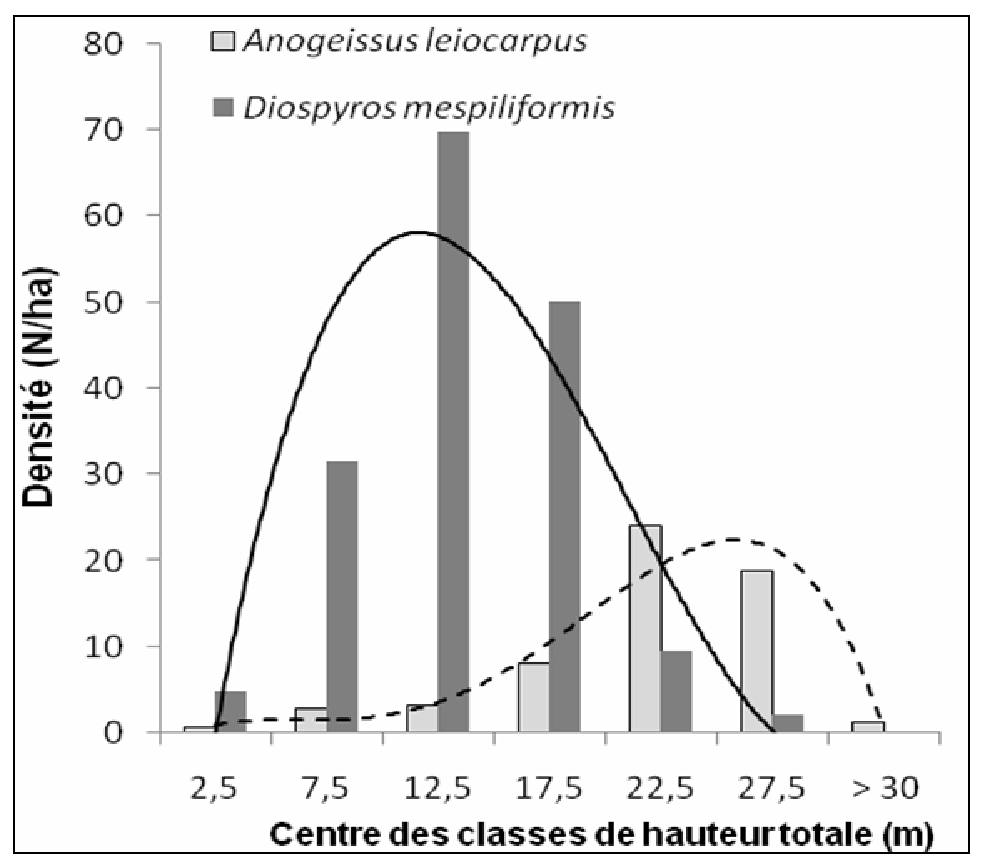

Figure 8: Structure verticale de D. mespiliformis et A. leiocarpus de la RFA.

fortement le diamètre et la hauteur moyens des arbres (Sahu et al., 2007). Toutes ces données constituent des arguments en faveur de la préservation des fonctions des écosystèmes et de la conservation de la biodiversité des forêts denses sèches (Principe 2, Critère 2.2 et Critère 2.3 (Boyle et al., 1998) ou Critère 5 (OAB-OIBT, 2003). Elles décrivent également des éléments de la biodiversité et fournissent des informations sur la mise en œuvre des directives de gestion pour la sauvegarde de cette aire protégée. Toutes ces informations présentées dans cet article sont importantes pour assurer la gestion durable des forêts (changement des superficies forestières, perte des communautés végétales associées aux forêts, perturbation de la structure forestière, perturbations dues aux feux de brousse, pression humaine) (Noss, 1999).

En terme de gestion, les méthodes utilisées jusqu'à une époque récente se caractérisaient par une approche basée sur la répression et le monopole du gouvernement central (contrôle strict, protectionnisme, exclusion des populations locales, interdiction des utilisations traditionnelles de la faune et de la flore). Les populations riveraines qui contestaient ces pratiques de gestion ont envahi la réserve à la faveur des troubles socio-politiques des années 90 que le pays a traversés. Les populations réinstallées dans la réserve et celle des villages environnants, y pratiquent l'agriculture et fabriquent d'importantes quantités de charbon de bois à partir des ressources ligneuses de la réserve. Ces activités ont contribué à la dégradation des formations forestières entre les années 1991 et 2000. Des réactions similaires des populations riveraines d'aires protégées ont été signalées dans d'autres pays notamment en Côte d'Ivoire dans la forêt classée de Monogaga (Adou Yao et N'guessan, 2006), dans le Parc de la Marahoué (Dibi N'da et al., 2008) ou encore dans la réserve forestière de Laf au Cameroun (Fotsing et al., 2002). Ainsi, les populations victimes des expropriations lors de la mise en place de la plupart des aires protégées se manifestent par des coupes anarchiques de bois, des défrichements illicites, une réinstallation illégale dans les villages jadis déplacés et la destruction 
massive de la faune sauvage (Tchamiè, 1994 ; Mengue-Medou, 2002) bafouant ainsi le Principe 3, Critère 3.1, 3.2 et 3.3 (Colfer et al., 1998). Plusieurs aires protégées du Togo se sont retrouvées dans les mêmes circonstances que la RFA (Adjonou et al., 2009). Compte tenu de ces erreurs, il apparaît indispensable de repenser les approches de gestion de l'ensemble des aires protégées du Togo qui doivent concilier conservation et satisfaction des populations locales. En effet, plusieurs auteurs ont montré que le succès de la gestion communautaire des forêts implique une effective prise de décision concertée (Sokpon and Agbo, 1999; Sheppard and Meitner, 2005). La première démarche dans ce sens consisterait à la sécurisation de cette aire protégée, c'est-à-dire, matérialiser effectivement les limites périphériques de la réserve (Principe 2, Critère 2.2 (OAB-OIBT, 2003)) afin d'éviter des intrusions des populations pour l'installation de nouvelles cultures.

\section{Conclusion}

L'analyse de la couverture forestière de la RFA à partir des images satellites révèle un taux annuel de régression de 3\%. L'analyse diachronique de la couverture végétale de la RFA a permis d'établir un zonage ou unités d'aménagement constituées de forêts denses sèches et galeries forestières (environ $10 \%$ de la superficie totale), de savanes boisées/arborées $(65 \%)$, de savanes arbustives (15\%) et de jachères. Les caractéristiques floristiques et structurales montrent que ces îlots de forêts denses sèches renferment 69 espèces ligneuses réparties en 62 genres et 23 familles. Ces espèces présentent une répartition régulière, traduisant leur régénération constante dans le temps. Toutefois, la capacité de restauration de ces forêts à partir de la régénération naturelle est faible. Toutes les informations présentées dans cet article sont importantes pour assurer la gestion durable des forêts (changement des superficies forestières, perte des communautés végétales associées aux forêts, perturbation de la structure forestière, perturbations dues aux feux de brousse, pression humaine). Compte tenu des erreurs passées de l'administration forestière, il apparaît indispensable de repenser les approches de gestion de la RFA et de façon générale de l'ensemble des aires protégées du Togo.

\section{REMERCIEMENTS}

Nous remercions l'Organisation Internationale des Bois Tropicaux (OIBT) qui a financé ce projet de recherche dans le cadre du programme de bourses pour le renforcement de capacités.

\section{BIBLIOGRAPHIE}

Adjonou K, Bellefontaine R, Kokou K. 2009. Les forêts claires du Parc National OtiKéran au Nord-Togo: structure, dynamique et impacts des modifications climatiques récentes. Revue Electronique Sécheresse, 20(4): 1 - 10.

Adou Yao CY, N'Guessan EK. 2006. Diversité floristique spontanée des plantations de café et de cacao dans la forêt classée de Monogaga, Côte d'Ivoire. Schweizerische Zeitschrift für Forstwesen, 157(2): 31 - 36.

Adou Yao CY, Blom EC, Dengueadhé KTS, Rompaey RSAR van, N'Guessan EK, Wittebolle G, Bongers F. 2005. Diversité floristique et végétation dans le Parc National de Taï, Côte d'Ivoire. Tropenbos-Côte d'Ivoire Serie 5 : 92.

Barbier C. 2004. Désertification et Forêts. Silva-Riat: Paris.

ATO-ITTO 2003. Principles, criteria and indicators for the sustainable management of African natural tropical forests. ITTO Policy Development Series 14, Yokohama, Japon, p. 28.

Bellefontaine R. 1997. Synthèse des espèces des domaines sahélien et soudanien qui se multiplient naturellement par voie végétative. Actes de l'Atelier “ Fonctionnement et gestion des écosystèmes forestiers contractés sahéliens, Niamey, nov. 1995", John 
Libbey Eurotext (ed) Orstom - Cirad Min. Agr. Niamey: 95 - 104.

Boyle TJB, Lawes M, Manokaran N, Prabhu R, Ghazoul J, Sastrapadja S, Thang HC, Dale V, Aeley H, Finegan B, Soberon J, Stork NE. 1998. Criteria and Indicator for Assessing the Sustainability of Forest Management: A Practical Approach to the Assessment of Biodiversity. Not published document, CIFOR, Bogor.C.J.P.

Colfer MA Brocklesby C, Diaw P, Etuge M, Guenter E, Harwell C, McDougall NM, Porro R, Porro R, Prabhu A, Salim MA, Sardjono AM, Tiani B, Tchikangua R. Vadley JW. 1998. Basic Assessment Guide for human Well-being. Preliminary Version, CIFOR, Bogor.

Curtis JT, Macintosh RP. 1950. The interrelations of certain analytic and synthetic phytosociological characters. Ecology, 31(3): 435 - 455

Dibi N'da H, N'guessan KE, Wadja ME, Affian K. 2008. Apport de la télédétection au suivi de la déforestation dans le Parc national de la Marahoué (Côte d'Ivoire); Revue Télédétection, 8(1): 17 - 34 .

Djiwa O. 2008. Dynamique forestière et diagnostic de la Forêt Classée d'Abdoulaye au Togo. Mém. Master, Agro ParisTech, ENGREF de Montpellier, p. 55.

FAO 1981. Manuel d'inventaire forestier. Etude FAO-Forêt $\mathrm{n}^{\circ} 31$, Rome ; 62.

Fongnzossie FE, Tsabang N, Nkongmeneck BA, Nguenang GM, Auzel P, Christina E, Kamou E, Balouma JM, Apalo P, Mathieu $H$, Valbuena $M$, Valère $M$. 2008. Les peuplements d'arbres du Sanctuaire à gorilles de Mengamé au sud Cameroun. Tropical Conservation Science, 1(3): 204 - 221.

Fotsing E, Ntoukpa M, Boubaoua A. 2002. Etat de la réserve forestière de Laf et des zones riveraines: Orientations d'aménagement et gestion de l'espace.
Actes du colloque, 27-31 mai 2002, Garoua, Cameroun

Gate U, Joshi NV, Gadgil M. 1998. On the patterns of tree diversity in the Western Ghats of India. Currents Sciences, 75 : 594 - 603.

Hall JB, Swaine MD. 1981. Distribution and ecology of vascular plants in a rain forest vegetation in Ghana. Geobotany I, p. 383.

Kokou K, Atato A, Bellefontaine R, Kokutse AD, Caballé G. 2006. Diversité des forêts denses sèches du Togo. Revue d'Ecologie, 61(3): 225 - 246.

Kokou K. 1998. Les mosaïques forestières au sud du Togo: biodiversité, dynamique et activités humaines. Thèse de Doctorat de l'Université de Montpellier II, p. 140.

Koné M., Aman A, Adou Yao CY, Coulibaly L, et N'Guessan EK, 2007. Suivi diachronique par télédétection spatiale de la couverture ligneuse en milieu de savane soudanienne en Côte d'Ivoir; Revue Télédétection, 7(1-2-3-4): p. 433446.

Lebrun JP, Stock AL. 1991 - 1997. Enumération des plantes à fleurs d'Afrique tropicale. 4 volumes. Conservatoires et Jardin Botanique de la ville de Genève.

Lindenmayer DB, Franklin JF, Fischer J. 2006. General management principle and a checklist of strategies to guide forest biodiversity conservation, Biol. Conserv., 131: 433 - 445.

Lopez P. 2004. Formes d'exploitation forestière et analyse du potentiel des forêts secondaires sèches. Une étude de cas dans le Nord-Ouest de Madagascar Eschborn, p. 117.

Mama JV, Oloukoi J. 2003. Evaluation de la précision des traitements analogiques des images satellitaires dans l'étude de la dynamique de l'occupation du sol, Télédétection, 3(5): 429-441.

Mendoza GA, Prabhu R. 2003. Qualitative Multi-criteria Approaches to Assessing Indicators of Sustainable Forest 
Resource Management. Forest Ecology and Management, 174: 329 - 43.

Mengue-Medou C. 2002. Les aires protégées en Afrique: perspectives pour leur conservation. VertigO - la Revue Electronique en Sciences de l'Environnement, 3(1), URL: http://vertigo.revues.org/index4126.html

Miles PD. 2002. Using biological criteria and indicators to address forest inventory data at the state level. For. Ecol. Manage., 155: 171 - 185.

Noss RF. 1999. Assessing and monitoring forest biodiversity: a suggest framework and indicators. For. Ecol. Manage., 115: $135-146$.

Pontius JRG. 2000. Quantification error versus location in comparison of categorical maps. Photogrammetric Engineering and Remote Sensing., 8(66): 1011-1016.

Prabhu R, Colfer CJP, Shepherd G. 2005. Critères et indicateurs d'une gestion forestière durable: nouveaux résultats des recherches du CIFOR au niveau de l'unité de gestion forestière. $O D I$, Document RDFN, 23a: 1 - 31.

Rasatatsihoarana HTF. 2007. Reconnaissances écologiques des aires forestières dans le Menabe sud en vue d'une délimitation de nouvelles aires protégées; Rapport final Atelier de Joachim (5 septembre 2007).

Sahu SC, Dhal NK, Reddy SC, Pattanaik C, Brahmam M. 2007. Phytosociological study of tropical dry deciduous forest of Boudh district, Orissa, India; Research Journal of Forestry, 2: 66 - 72.

Sheppard SRJ, Meitner M. 2005. Using multicriteria analysis and visualisation for sustainable forest management planning with stakeholder groups, For. Ecol. Manage, 207: 171 - 187.
Sokpon N, Biaou SH, Ouinsavi C, Hunhyet O. 2006. Bases techniques pour une gestion durable des forêts claires du NordBénin: rotation, diamètre minimal d'exploitabilité et régénération. Bois et Forêts des Tropiques, 287: 45 - 57.

Sokpon N, Agbo V. 1999. Sacred groves as tools for indigenous forest management in Benin. Annales des Sciences Agronomiques Univ. Nat du Bénin, 1: $162-175$.

Sundarapandian SM, Swamy PS. 1997. Plant biodiversity at low-elevation evergreen and moist deciduous forests at Kodayar (Western Ghats, India). Int. Journ. Ecol. Environ. Sci., 23: 363 - 379.

Tchamiè KTT. 1994. Enseignements à tirer de l'hostilité des populations locales à l'égard des aires protégées au Togo. Unasylva, 176: $22-27$.

Trochain JL. 1957. Accord interafricain sur la définition des types de végétation de l'Afrique tropicale. Bull. Int. d'Etudes Centrafricaines, 13 - 14: 556 - 593.

Vanpraet CL. 1980. Projet pilote sur la surveillance continue de la couverture forestière tropicale, cartographie $\mathrm{du}$ couvert végétal et étude de ces modifications, Togo. FAO/PNUE, Rome.

White F. 1986. La végétation d'Afrique. Mémoire accompagnant la carte de végétation de l'Afrique. UNESCO/AETFA/UNSO.

Zapfack L. 2005. Impact de l'agriculture itinérante sur brûlis sur la biodiversité végétale et la séquestration du carbone. Thèse d'Etat, Université de Yaoundé I. 